\title{
Extinction of Behavior in Infant Rats: Development of Functional Coupling Between Septal, Hippocampal, and Ventral Tegmental Regions
}

\author{
H. P. Nair and F. Gonzalez-Lima \\ Institute for Neuroscience and Department of Psychology, University of Texas at Austin, Austin, Texas 78712
}

Learning of a behavior at a particular age during the postnatal period presumably occurs when the functional brain circuit mediating the behavior matures. The inability to express a learned behavior, such as inhibition, may be accounted for by the functional dissociation of brain regions comprising the circuit. In this study we tested this hypothesis by measuring brain metabolic activity, as revealed by fluorodeoxyglucose (FDG) autoradiography, during behavioral extinction in 12- and 17-dold rat pups. Subjects were first trained on a straight alley runway task known as patterned single alternation (PSA), wherein reward and nonreward trials alternate successively. They were then injected with FDG and given 50 trials of continuous nonreward (i.e., extinction). Pups at postnatal day 12 (P12) demonstrated significantly slower extinction rates compared to their P17 counterparts, despite the fact that both reliably demonstrated the PSA effect, i.e., both age groups

The presence or absence of a behavior during the postnatal period presumably reflects the integrity of the functional brain circuit supporting it. As a behavior emerges over the course of the postnatal period, it may be inferred that the neural systems and, importantly, the functional interactions between neural systems that support the behavior are maturing. By analyzing brain functional activity during transitional periods of behavioral development, we may gain valuable insight into ontogenetic changes in the integrative properties of the brain.

The ability to suppress a behavioral response is a relatively late-appearing phenomenon in altricial mammalian species. Developing rats, for example, show impairments on passive avoidance (Myslivecek and Hassmannova, 1991) and habituation tasks (Bronstein et al., 1971; Feigley et al., 1972) well into the third postnatal week. Likewise, we recently demonstrated that preweanling rat pups trained in a straight alley runway on a schedule of alternating reward and nonreward, known as patterned single alternation (PSA), show different response suppression rates when switched to continuous nonreward (i.e., behavioral extinction): whereas postnatal day 16 (P16)-P17 pups immediately suppress responding during extinction, P11-P12

Received May 7, 1999; revised June 17, 1999; accepted July 20, 1999

This work was supported by National Institutes of Health Grants RO1 NS37755 to F.G.L. and F31 MH11968 to H.P.N. We thank Professor Abram Amsel for providing his facilities for the behavioral work, and we dedicate this paper to him on the occasion of his retirement.

Correspondence should be addressed to Dr. F. Gonzalez-Lima, Behavioral Neuroscience, Mezes Hall 330, University of Texas at Austin, Austin, TX 78712.

Copyright (C) 1999 Society for Neuroscience $0270-6474 / 99 / 198646-10 \$ 05.00 / 0$ distinguished between reward and nonreward trials during acquisition. Covariance analysis revealed that the dentate gyrus, hippocampal fields CA1-3, subiculum, and lateral septal area were significantly correlated in P17 but not P12 pups. Significant correlations were also found between the lateral septal area, ventral tegmental area, and the medial septal nucleus in P17 pups. Similar correlative patterns were not found in P12 and P17 handled control animals. Taken together, these results suggest that septal, hippocampal, and mesencephalic regions may be functionally dissociated at P12, and the subsequent maturation of functional connectivity between these regions allows for the more rapid expression of behavioral inhibition during extinction at P17.

Key words: brain imaging; hippocampus; covariance; extinction; development; rat

pups demonstrate significantly slower extinction rates relative to their older counterparts (Lilliquist et al., 1999).

In this study, we combined fluorodeoxyglucose (FDG) autoradiography, quantitative image analysis, and covariance analysis to study the development of functional interactions in this agerelated change in extinction behavior. FDG, a radiolabeled glucose analog, can be used to measure regional changes in brain metabolic activity occurring during behavior. Because energy utilization and functional activity are closely correlated (Sokoloff, 1992), FDG serves as an index of brain functional activity. When combined with interregional covariance analysis, regional interactions within and between neural systems that occur under a particular experimental condition may be determined. Interregional covariance analysis has been applied previously to various measurements of neuronal activity to study functional interactions during cognitive tasks (Horwitz et al., 1992a), in disease states (Horwitz et al., 1987), and injury (Horwitz et al., 1992b).

Previous reports have indicated that the septohippocampal system is involved in extinction behavior, because adult rats with septal (Henke, 1977) and hippocampal lesions (Winocur and Mills, 1969; Rawlins et al., 1980; Diaz-Granados et al., 1992) demonstrate persistent responding during extinction after appetitive runway training. Based on these data and given the dynamic structural and physiological changes in the hippocampus occurring during this age period, we hypothesized that there would be developmental changes in the functional relationships between hippocampal subfields, hippocampal subfields and the septal area, as well as between the septohippocampal system and other regions that might be involved in supporting rapid extinction. These other regions include the hypothalamus and the ventral tegmental 


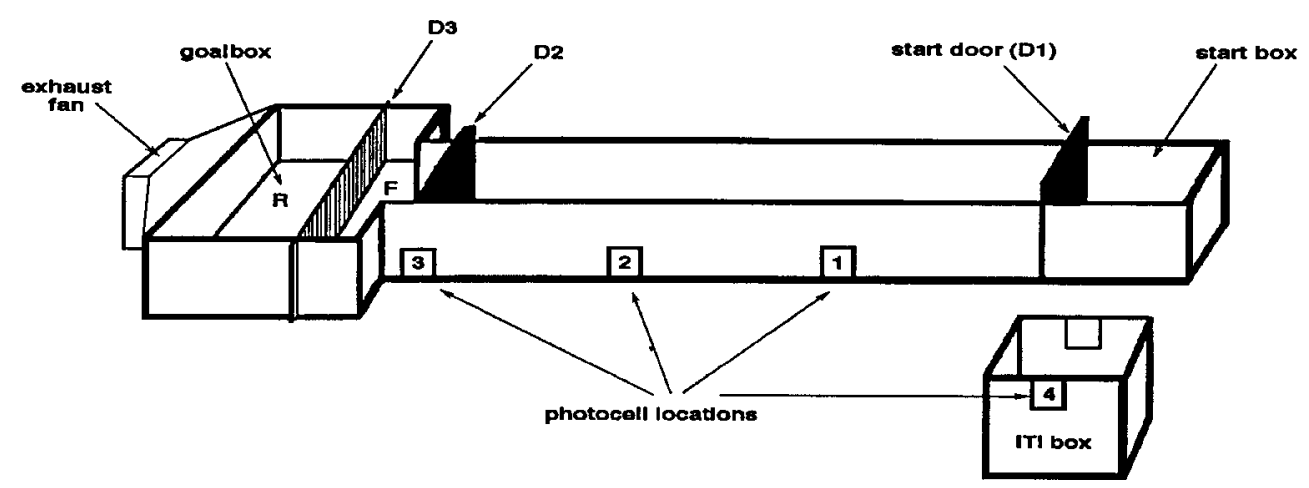

Figure 1. Schematic view of pup runway. Photocells are placed at positions 1,2 , and 3 , to time start, run, and goal speeds and intertrial intervals. D1, Door from startbox to alley; $D 2$, door from alley to goalbox; $D 3$, automated door, dividing goalbox into two compartments, which is controlled by signals from Photocell 3 . An anesthetized dam placed in the rear compartment $(R)$ is accessible on rewarded trials when D3 is raised. On nonrewarded trials, pups remain in the front compartment $(F)$ with D3 in lowered position. On rewardmilk trials, milk is delivered via an infusion pump (not shown) into the oral cannula of the pup while it is attaching to the nipple and suckling. The length of the runway is adjusted depending on age group. For 16- to 17 -d-old pups, the length is $60 \mathrm{~cm}$ from start door to goal box. The length is shortened to $45 \mathrm{~cm}$ for $11-$ to 12-d-old pups.

area, regions previously implicated in rewarded behavior (Gonzalez-Lima et al., 1993; Kosobud et al., 1994). Four groups were used in this study: two age groups that were trained on PSA and then extinguished, and a handled control group within each age. The results demonstrate differences in functional relationships among the aforementioned regions between trained age groups and between trained and control animals at each age.

\section{MATERIALS AND METHODS}

Twenty-four rat pups of the Holtzman strain, aged P11-P12 or P16-P17 and raised in our colony at the Animal Resource Center at the University of Texas at Austin were used. The day of birth was designated as P0. All experimental procedures were approved by the University of Texas Institutional Animal Care and Use Committee and conform to all Federal and National Institutes of Health guidelines. Four groups of animals were used: two trained groups at each age (PSA17 and PSA12) and two handled control groups ( $\mathrm{HC} 17$ and $\mathrm{HC} 12$ ). The handled controls served to control for brain activation not related to the behavioral training. There were seven subjects in each trained group and five subjects in each control group.

Behavioral training. All training was conducted in the straight-alley runway pictured in Figure 1.

On the day before training (day 0), pups from separate litters were fitted with an oral cannula and familiarized with the training apparatus. On days 1 and 2, pups were either trained on PSA or handled for 200 trials (five sessions of 40 trials each across $2 \mathrm{~d}$ ). After acquisition of PSA or handling, all subjects were injected with FDG and given one reward trial followed by 49 nonreward (extinction) trials. The intertrial interval (ITI) for both acquisition and extinction trials was $8 \mathrm{sec}$.

Acquisition. During PSA training, animals were first placed in the start box (Fig. 1). The start box door was then lifted, and the pup was allowed to traverse the runway. Photocells were used to record running speed. On nonreward $(\mathrm{N})$ trials, the goal gate remained in place, confining the subject to the anterior chamber of the goalbox. On reward (R) trials, the goal gate was automatically released to allow the subject access to an anesthetized dam. After attaching to a nipple and suckling, the pup was rewarded with milk-diet infusion directly into its mouth via the chronic oral cannula. Between trials, the subject was placed in the ITI box for 8 sec. Handled control animals were given the amount of reward they would have received in a 40 trial acquisition session but outside of the runway apparatus. They were then placed in and out of the runway for 20 trials. This was repeated for each acquisition session.

Extinction. All animals were injected with $18 \mu \mathrm{Ci} / 100 \mathrm{gm}$ FDG and then extinguished (PSA animals) or placed in and out of the runway (handled controls) for 50 trials. The first trial of the extinction session was a reward trial.

Reward schedules and goal gate were controlled by a Fortran program running on an IBM AT computer (Lilliquist et al., 1999). Running speeds were recorded by the same computer for later analysis.

FDG autoradiography. The FDG protocol of Gonzalez-Lima (1992) was used. Immediately before the extinction session (session 6), subjects were injected intraperitoneally with $18 \mu \mathrm{Ci} / 100 \mathrm{gm}$ of body weight of $\left[{ }^{14} \mathrm{C}(\mathrm{U})\right]$ FDG; (specific activity, $300 \mathrm{mCi} / \mathrm{mmol}$; American Radiola- beled Chemicals, St. Louis, MO) in $0.1 \mathrm{ml}$ of physiological saline. Animals were trained for $\sim 50 \mathrm{~min}$, the time it takes to complete the extinction session. After completion of the test period, the animal was removed from the chamber and rapidly decapitated. The brain was then quickly removed and frozen in $-40^{\circ} \mathrm{C}$ isopentane for $\sim 2-3 \mathrm{~min}$. Sections of the brain at $40 \mu \mathrm{m}$ were taken in a cryostat at $-20^{\circ} \mathrm{C}$ (Reichert-Jung 2800 Frigocut E). Slices used for FDG were picked up on slides and immediately dried on a hot plate at $60^{\circ} \mathrm{C}$.

The FDG slides were apposed to Kodak (Eastman Kodak, Rochester, NY) EB-1 film and placed inside Kodak X-O-Matic cassettes for 2 weeks. Plastic microscale standards of known ${ }^{14} \mathrm{C}$ concentrations (Amersham, Arlington Heights, IL) were placed with each film. The standards were used to calculate ${ }^{14} \mathrm{C}$ concentrations $(40-1069 \mathrm{nCi} / \mathrm{gm}$ of tissue). Films were developed in Kodak D-19 for 2 min, rinsed in 2\% acetic acid for $1 \mathrm{~min}$, and fixed for $8 \mathrm{~min}$. Selected sections were stained with cresyl violet after autoradiographic exposure to delineate regions morphologically.

Quantitative image analysis. Incorporation of FDG was quantified using JAVA image analysis software (version 1.4; Jandel Scientific, Corte Madera, CA). Images from the film were placed on a DC-powered light box and captured through a black and white video camera (Javelin JE2362). The analog signal from the camera was transmitted to a frame grabber (Targa M-8) mounted in an Everex 486/25 computer where the image is digitized. The image was corrected for film background and optical distortions from the camera through subtraction of the background. A calibration curve was created based on the absolute gray levels of the ${ }^{14} \mathrm{C}$ standards on the film. Subsequent densitometric measures taken from brain images were then automatically expressed in terms of isotope incorporation per gram of tissue (nanocuries per gram).

Regions of interest. FDG incorporation was measured in 11 different regions: dentate gyrus, CA1 and CA3 fields, and subiculum of the hippocampus; entorhinal cortex; medial and lateral septum; ventral tegmental area; lateral hypothalamus; mammillary bodies; and primary motor cortex. The atlas of Sherwood and Timiras (1970) was used to delimit the regions measured. Approximate sampling levels with anatomical coordinates (relative to the interaural line) for each age group are presented in Figure 2.

Measures from each brain region of interest were taken from at least three adjacent sections, and four adjacent readings covering each area were taken in each section. The value for each brain area from each subject was then computed as the mean of all readings from the three adjacent sections. To help reduce variation in the intensity of the FDG labeling that is unrelated to the experimental manipulation (a potential source for spurious correlations; see Horwitz et al., 1992b), ${ }^{14} \mathrm{C}$ values from each brain area were divided by the average ${ }^{14} \mathrm{C}$ value for the whole brain of each animal (whole-brain ratio). Whole brain averages were obtained by taking the mean of the average isotope incorporation for each section of the brain. Whereas raw values were used to evaluate mean differences in FDG uptake, whole brain ratios were used for the covariance analysis.

Statistical analysis. For analysis of behavioral data, PSA acquisition and extinction trials were combined into five trial blocks. Behavioral effects were evaluated using repeated measures ANOVA followed by Neuman-Keuls post hoc tests. Repeated measures ANOVA was also 


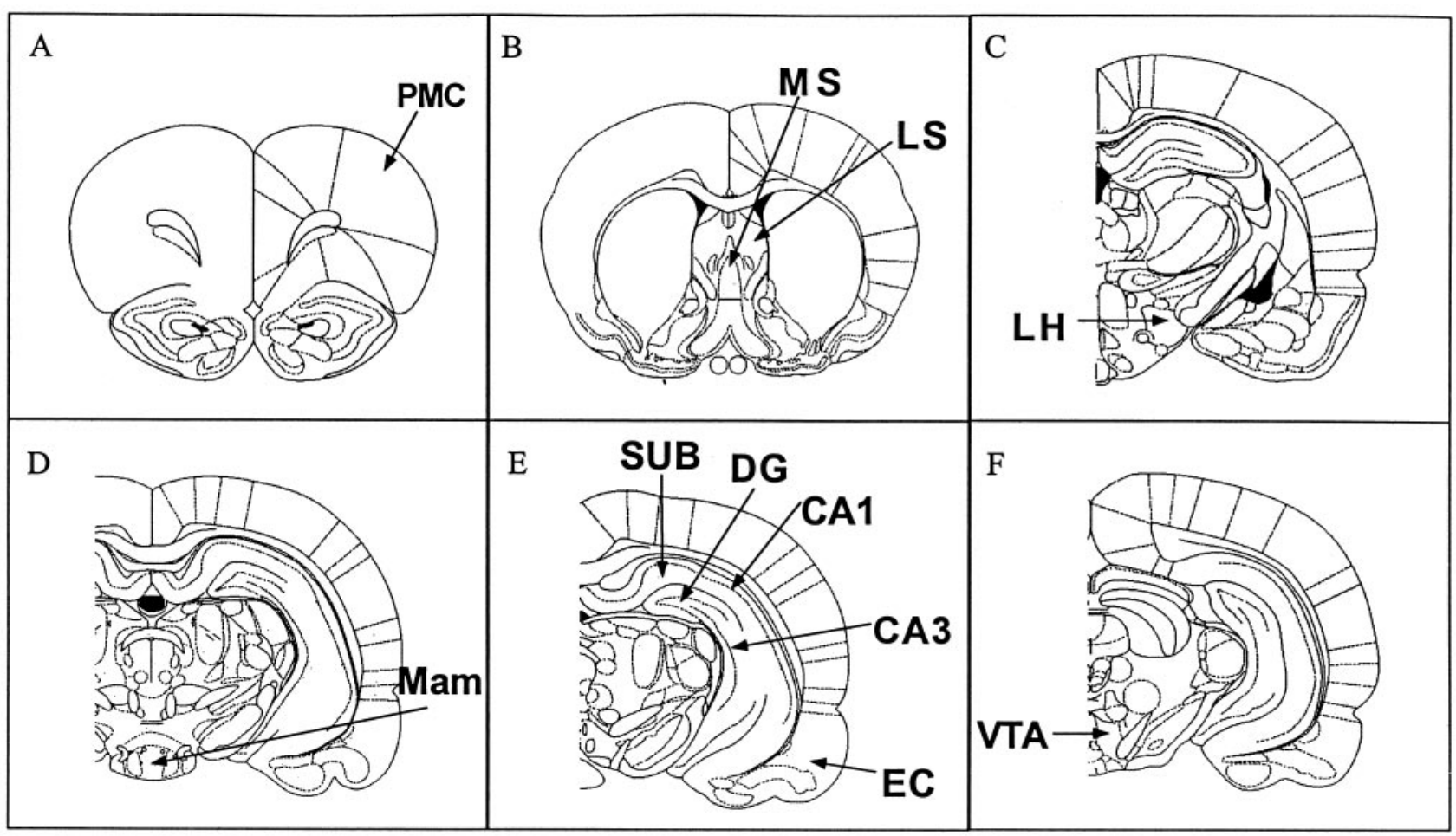

Figure 2. Sampled regions. Regions of interest were from six different anatomical levels. The distance from interaural line for each age group is as follows (in millimeters): $A$, $\mathrm{P} 12,7.0 ; \mathrm{P} 17,9.0 ; B, \mathrm{P} 12,5.3 ; \mathrm{P} 17,6.5 ; C, \mathrm{P} 12,2.6 ; \mathrm{P} 17,3.2 ; D, \mathrm{P} 12,1.2 ; \mathrm{P} 17,1.6 ; E, \mathrm{P} 12,0.8 ; \mathrm{P} 17,1.2 ; F, \mathrm{P} 12,0.4 ; \mathrm{P} 17,1.2$. $P M C$, Primary motor cortex; $M S$, medial septum; $L S$, lateral septum; $L H$, lateral hypothalamus; Mam, mammillary bodies; Sub, subiculum; $D G$, dentate gyrus; EC, entorhinal cortex; VTA, ventral tegmental area. (Section diagrams were reproduced with permission from The Rat Brain in Stereotaxic Coordinates, G. Paxinos and C. Watson, New York: Academic, 1997, CD-ROM.)

used to compare mean percent differences between reward and nonreward trials across blocks and ages. Percent differences between reward and nonreward trials were computed with the formula $((R-N) / R) \times$ 100 , where $R=$ reward trials and $N=$ nonreward trials.

Regional changes in FDG incorporation between PSA and handled controls at each age were evaluated using repeated measures ANOVA followed by tests for simple effects. For the interregional covariance analysis, Pearson product moment correlations were computed between all measured regions for each group. To ensure the reliability of correlations, all correlations were subjected to the jackknife procedure, whereby each individual is sequentially removed, and correlations are computed on a dataset with $n-1$ subjects. Correlations were considered to be "reliably" significant if they remained significant $(p<0.05)$ throughout all iterations. This methodology ensures that a significant correlation is not an artifact arising from an outlying subject, a problem that can arise with small sample sizes. Having obtained significant correlations, they were transformed to $z$ scores, and omnibus planned groups comparisons were made using a Student's $t$ test for paired data. The rejection level was set at a two-tailed probability above 0.05 , after correction for multiple comparisons using a modified Bonferroni correction procedure (Hochberg, 1988).

Neurobehavioral correlations were used to correlate average extinction rate against FDG activity in the regions of interest for all PSA rat pups. The average extinction was computed with the formula $((I-F) / 50$ $\min ) \times 100$, where $I$ is the initial speed (run speed on block 1 ), $F$ is the final speed (run speed on block 10), and $50 \mathrm{~min}$ is the approximate time of the 10 blocks of extinction training.

\section{RESULTS}

\section{Behavioral tests}

Throughout behavioral training, overall run speed was substantially lower in P12 animals as compared to P17 animals, because of the P12 group's limited ability to run faster (their ventral side is in contact with the floor, compromising their running ability). Therefore, run speeds during acquisition were converted to per- centage of maximum run speed on rewarded trials for both age groups. Analysis of reward versus nonreward trials for each PSA trained group was then performed on both converted and raw values. In both cases, after a significant repeated measures ANOVA, post hoc comparisons revealed significant differences $(p<0.05)$ between rewarded and nonrewarded trials on blocks 10-12 and 15-20 for P16-P17 pups (Fig. 3A) and 10-12, 14-16, and 18-20 for P11-P12 pups (Fig. 3B). Hence, both P16-P17 and P11-P12 groups successfully acquired PSA, because both discriminated between reward and nonreward trials on roughly half of the blocks of trials during the latter half of training.

Repeated measures ANOVA of percentage difference between run speed on reward and nonreward trials on the last three blocks of training (i.e., last 30 trials of acquisition) revealed no significant difference between blocks, suggesting that running times of PSA17 and PSA12 rats had stabilized by the end of acquisition training. In the final three blocks, a significant main effect for age $(p<0.05)$ was found, indicating that the final difference in run speeds between reward and nonreward trials were greater in the older group. However, the average percentage difference between reward and nonreward run speeds across all five sessions did not differ between ages (38\% for PSA17 and 34\% for PSA12 pups).

Statistical analyses of extinction rates were performed on run speeds expressed as percentage of terminal acquisition speed (last block of acquisition) on rewarded trials. Repeated measures ANOVA of extinction rates revealed significant differences in run speeds between P12 and P17 animals. Subsequent post hoc analyses revealed significantly $(p<0.05)$ higher run speeds in $\mathrm{P} 12$ pups on blocks 3 and $4-10$. Thus, both P16-P17 and P11-P12 pups successfully acquired the PSA discrimination, yet the 
A

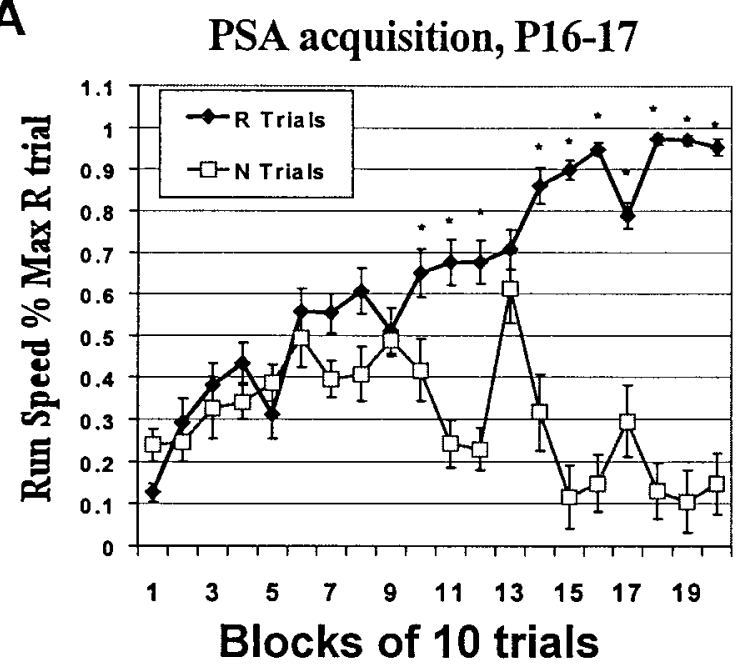

B

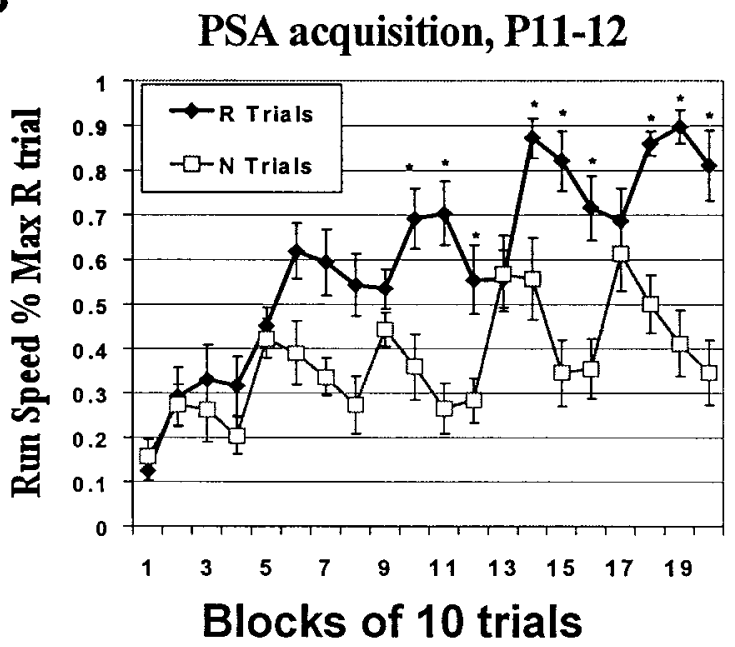

Figure 3. Acquisition data. The faster run speeds (presented as a percentage of maximum run speed on $\mathrm{R}$ trials) on rewarded trials by the end of training indicate that both $\mathrm{P} 17(A)$ and $\mathrm{P} 12(B)$ successfully discriminated between reward $(R)$ and nonreward $(N)$ trials. *Indicates significant difference $(p<0.05)$ between reward and nonreward trials.

younger group demonstrated attenuated extinction rates relative to their older counterparts (Fig. 4).

Although we did not quantify behavioral activity in control animals, we noted that these animals engaged in exploratory behavior (traversing runway, rearing) throughout extinction training, and substantially more so in the first two or three blocks than in later blocks. Qualitatively, the degree of motor activity occurring across blocks in the control animals was comparable to that of trained groups.

\section{Regional FDG uptake}

Table 1 presents mean FDG values with SEs.

\section{Means analysis}

Repeated measures ANOVA of regional FDG values (with region operating as the within subject factor) revealed no differences between PSA and handled control animals at either age. Thus, FDG values for trained and control groups at each age were pooled, and age-related differences in FDG values for each region were evaluated. Repeated measures ANOVA followed by

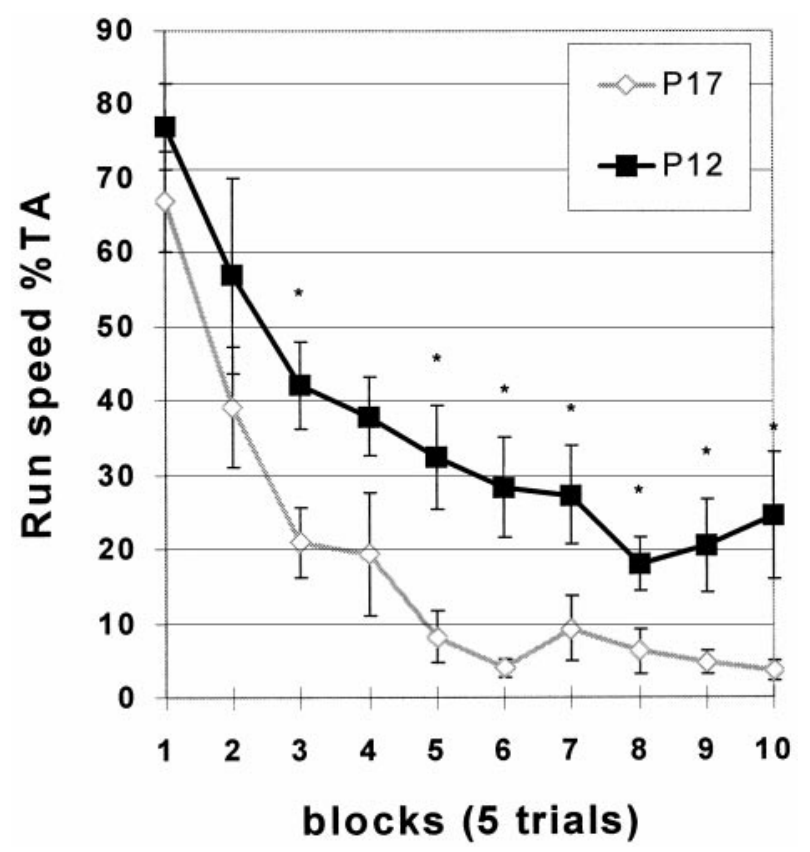

Figure 4. Extinction data. P12 pups demonstrated attenuated extinction rates (presented as percentage of maximum run speed on the last block of rewarded trials during acquisition) on trials 3 and 5-10. Asterisk indicates significant difference $(p<0.05)$ between $\mathrm{P} 12$ and $\mathrm{P} 17$ groups.

tests for simple effects revealed significantly higher $(p<0.05)$ FDG values in the mammillary bodies, subiculum, CA1 and CA3 fields, medial septum, and VTA in P17 animals as compared to P12 (Fig. 5). Figure 6 illustrates this age-related increase in FDG uptake in the hippocampal formation.

\section{Covariance analysis}

In general, the $r$ values obtained for pairwise correlations tended to be positive across all four groups (Tables 2, 3). There were 90 correlations $<0.5$ and $130>0.5$, so there were similar total numbers of low and high correlations. However, the PSA17 group was characterized by very high correlations.

Covariance analysis of FDG data revealed significant positive correlations $(p<0.05)$ between CA1 and subiculum, lateral septum and subiculum, medial septum and subiculum, and dentate gyrus and lateral septum, in PSA12 pups (Table $3 A$, Fig. $7 C$ ). These correlations were not significant in the HC12 group, although a significant positive correlation was found between the PMC and VTA (Table 3B, Fig. 7D). In the PSA17 group, significant positive correlations were found between hippocampal subfields (dentate gyrus and CA3, CA3 and CA1, CA1 and subiculum), CA3 and lateral septum, subiculum and lateral septum, medial and lateral septum, lateral septum and VTA, medial septum and VTA, and mammillary bodies and lateral hypothalamus (Table $2 A$, Fig. $7 A$ ). Significant positive correlations were found between the lateral and medial septum, and VTA and lateral hypothalamus in the $\mathrm{HC} 17$ group (Table $2 B$, Fig. $7 B$ ).

Although there were trends for similar correlations in the PSA12 as found in PSA17 pups, these were not reliably significant, as determined by the jacknife procedure. This procedure rules out the possibility of an outlier influencing the correlation, which is particularly important for small sample sizes. Scatter plots of the correlations between the CA3 and DG, CA3 and LS, and CA1 and CA3 demonstrate the more linear nature of values 
Table 1. FDG values (mean $\pm \mathrm{SE} ; \mathrm{nCi} / \mathrm{gm})$

\begin{tabular}{|c|c|c|c|c|}
\hline Structure & PSA17 & $\mathrm{HC} 17$ & PSA12 & $\mathrm{HC} 12$ \\
\hline Entorhinal cortex (EC) & $552.23 \pm 28.02$ & $564.24 \pm 46.80$ & $469.45 \pm 36.18$ & $495.01 \pm 63.03$ \\
\hline $\mathrm{CA} 1 *$ & $715.92 \pm 20.08$ & $747.96 \pm 71.54$ & $545.58 \pm 37.47$ & $556.24 \pm 25.18$ \\
\hline CA3* & $722.10 \pm 32.40$ & $710.76 \pm 60.17$ & $565.16 \pm 31.80$ & $556.29 \pm 35.46$ \\
\hline Dentate gyrus (DG) & $546.92 \pm 18.96$ & $576.84 \pm 57.24$ & $483.25 \pm 41.02$ & $426.94 \pm 32.39$ \\
\hline Subiculum $(\mathrm{Sub})^{*}$ & $889.61 \pm 33.51$ & $1009.92 \pm 92.10$ & $664.39 \pm 42.48$ & $674.94 \pm 54.79$ \\
\hline Medial septum (MS)* & $938.60 \pm 26.23$ & $776.25 \pm 84.35$ & $705.44 \pm 51.45$ & $683.95 \pm 49.09$ \\
\hline Lateral septum (LS) & $626.42 \pm 46.26$ & $652.47 \pm 67.4$ & $494.76 \pm 39.40$ & $559.89 \pm 37.36$ \\
\hline Lateral hypothalamus (LH) & $1183.68 \pm 73.88$ & $1198.02 \pm 111.32$ & $1077.78 \pm 83.47$ & $1213.98 \pm 160.70$ \\
\hline Mammillary bodies (Mam)* & $1280.58 \pm 82.47$ & $1278.68 \pm 90.86$ & $803.023 \pm 82.35$ & $868.45 \pm 190.74$ \\
\hline Ventral tegmental area (VTA)* & $837.10 \pm 45.44$ & $916.40 \pm 73.10$ & $708.29 \pm 61.95$ & $746.70 \pm 125.97$ \\
\hline Primary motor cortex (PMC) & $1121.15 \pm 78.36$ & $1174.94 \pm 133.51$ & $1100.36 \pm 39.65$ & $896.18 \pm 52.63$ \\
\hline
\end{tabular}

${ }^{*} p<0.05$.

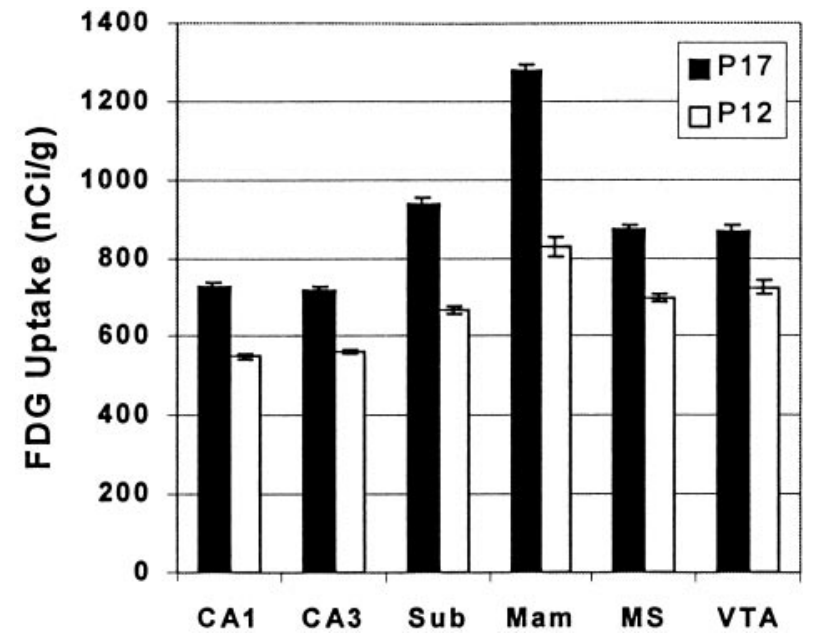

Figure 5. Pooled FDG uptake data. Of the eleven regions sampled, the CA1 and CA3 fields of the hippocampus, subiculum $(S u b)$, mammillary bodies (Mam), medial septum (MS), and ventral tegmental area (VTA), were significantly higher in P17 animals compared with P12.

in the PSA17 (Fig. $8 A$ ) group as compared to PSA12 (Fig. $8 C$ ), $\mathrm{HC17}$ (Fig. $8 B$ ), and HC12 (Fig. 8D) groups.

The correlations significantly different from zero in the PSA17 group were compared simultaneously with the corresponding values in the HC17 and PSA12 groups. The average correlation coefficients, after $z$ transformation, revealed that the PSA17 group had an average score $(1.77 \pm 0.12)$ greater than in both the HC17 $(0.82 \pm 0.21 ; \mathrm{df}=14 ; p=0.01)$ and PSA12 $(1.05 \pm 0.10 ; \mathrm{df}=14 ;$ $p=0.00003)$ groups. Although these same correlations were not all significantly different from zero in the PSA12 group, their mean value was higher than the same correlations in the $\mathrm{HC} 12$ group $(0.57 \pm 0.17 ; \mathrm{df}=14 ; p=0.05)$.

Neurobehavioral correlations between extinction performance and FDG regional activity in the PSA animals revealed that activity in the CA1, CA3, subiculum, medial and lateral septum, and mammillary bodies were significantly correlated with performance (Table 4).

Taken together, these results show that 17 -d-old rat pups, after learning to discriminate between reward and nonreward trials during acquisition, readily inhibited responding when switched to extinction. FDG autoradiography, quantitative image analysis, and covariance analysis revealed significant positive correlations between hippocampal, septal, ventral tegmental, and hypotha- lamic regions in these animals (Fig. 7D). The neurobehavioral correlational analysis revealed that several of these regions were also correlated with performance during extinction, providing further support for their role in extinction behavior.

Despite discriminating between reward and nonreward during acquisition, P12 pups demonstrated attenuated extinction rates relative to P17 animals, as well as a different pattern of correlative activity among the sampled regions (Fig. 7B). Although there were trends in PSA12 pups for similar patterns of interregional covariation among the sampled regions, especially within the hippocampal subfields, they were not reliably significant.

Aside from positive correlations between the primary motor cortex and ventral tegmental area in the HC12 group, and positive correlations between the MS and LS and VTA and lateral hypothalamus in HC17 pups, there were no other significant correlations found in control groups, indicating that in general the covariance patterns found in PSA17 and PSA12 were specific to extinction behavior.

\section{DISCUSSION}

\section{Regional differences in FDG uptake}

Whereas functional relationships were different between sampled regions during extinction training relative to the control situation for each age group, the net energy consumption remained the same between sampled regions. This is not an unusual situation and in part forms the basis for evaluating changes in covariance relationships in brain metabolic mapping data (Horwitz et al., 1987; McIntosh and Gonzalez-Lima, 1994).

Alternatively, there were developmental increases in FDG incorporation in the mammillary bodies, CA1, CA3, subiculum, medial septum, and VTA, regions that also showed changes in covariance patterns across PSA groups. Because changes in FDG activity usually reflect the energy requirements of the sodiumpotassium pump and specifically, its maintenance of ion concentrations after spike activity at nerve terminals (Gonzalez-Lima, 1992), the increases in incorporation are likely to reflect increases in the number of synapses and axonal and dendritic components in these regions. Heightened local cerebral glucose utilization has previously been reported to coincide with synaptic density in other species such as the cat (Chugani et al., 1991) and human (Chugani and Phelps, 1986).

The absence of similar increases in FDG activity in the EC, DG, lateral septum, lateral hypothalamus, and primary motor cortex is probably because of the particular ages assessed, because glucose utilization has previously been shown to increase sub- 


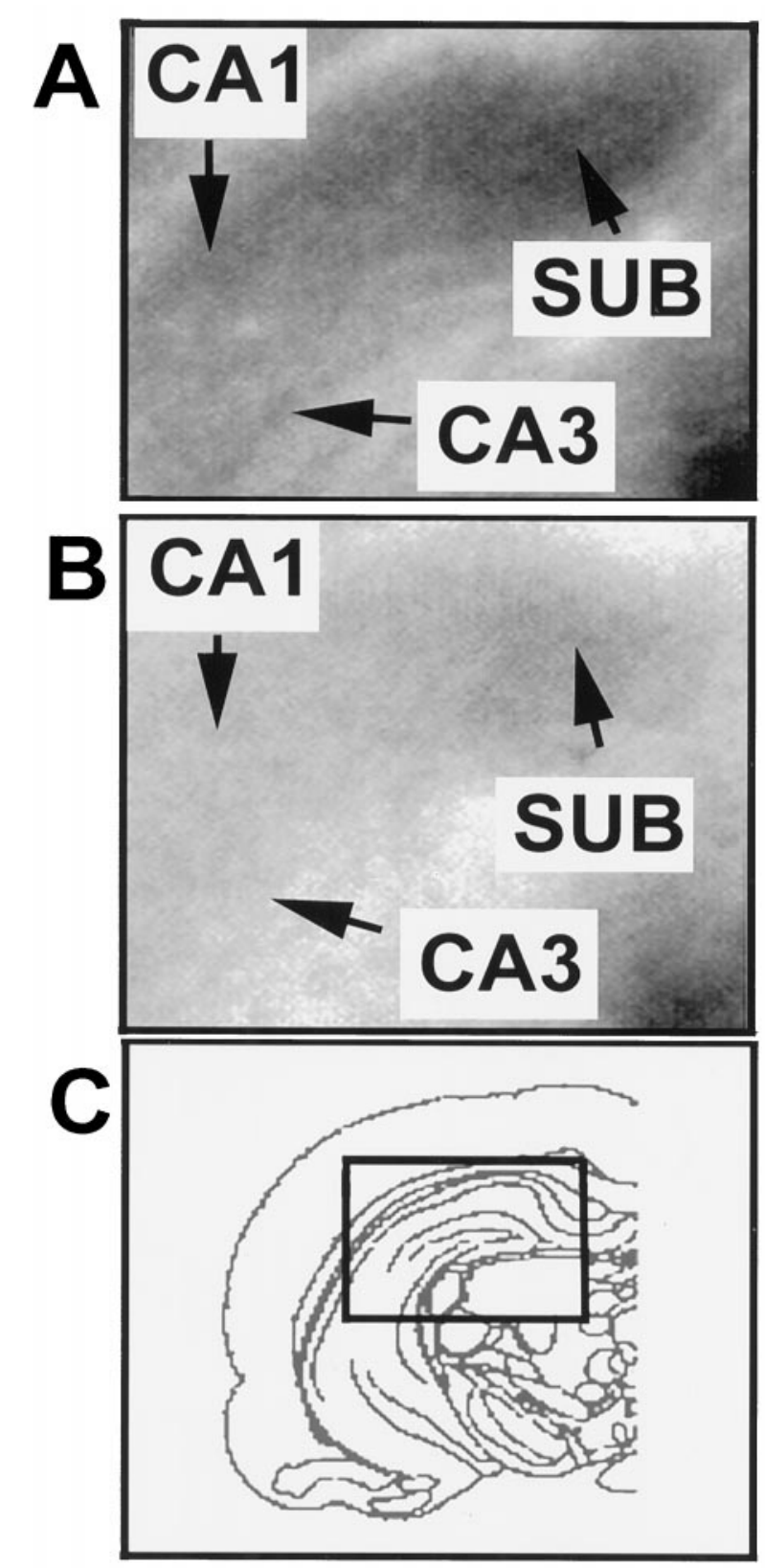

Figure 6. Autoradiographic images demonstrating changes in FDG uptake in CA1, CA3, and subiculum between $(A) \mathrm{P} 17$ and $(B) \mathrm{P} 12$ pups. A schematic of the region imaged is presented in $C$.

stantially in these regions between P17 and P21 in the rat (Nehlig et al., 1989). On the other hand, the same regions that show increases in glucose utilization here were previously reported by Nehlig et al. (1989) to be uniformly low and unchanged between $\mathrm{P} 10$ and P17. The primary difference between the two studies is that we measured FDG incorporation during behavioral activation (i.e., in animals either extinguishing an instrumental response or being handled), whereas Nehlig et al. (1989) measured glucose utilization under resting conditions. Hence, whereas low and uniform rates of glucose utilization in young rat pups may occur under resting conditions, energy consumption is characterized by regional heterogeneity during conditions of behavioral activation.

\section{Septohippocampal, midbrain, and hypothalamic interactions during extinction}

The covariance analysis approach assumes that during a particular behavior, if a functional network of brain regions is consistently activated across subjects, then intersubject variation in the degree of activation will result in highly correlated activity between pairs of regions comprising the network (Horwitz et al., 1992b). Our results indicate that in PSA17 pups, a functional network involving the septohippocampal system, VTA, lateral hypothalamus, and mammillary bodies is activated during extinction training and may underlie their immediate inhibition of instrumental responding. The same functional network does not appear to be operating in PSA12 pups, which show attenuated extinction rates, or in the handled control groups, which never engaged in instrumental behavior or the inhibition of it.

The changes in covariance relationships within the septohippocampal system of the PSA17 group corroborates previous reports indicating septohippocampal involvement in the extinction of an instrumental response. Lesions of the septum, hippocampus, or fornix lead to increased resistance to extinction after training, for example, on a continuous reinforcement schedule (where each trial is rewarded during acquisition) in adult (Kimble and Kimble, 1965; Winocur and Mills, 1969; Jarrad et al., 1986) as well as preweanling rats (Lobaugh et al., 1985; Amsel, 1992). The major connections of the hippocampal formation via the fornix are to the septal area (Linke and Frotscher, 1993; Linke et al., 1995) and the mammillary bodies (Sziklas and Petrides, 1998). These regions showed significant neurobehavioral correlations between the extinction rate and FDG uptake, providing support for their role in extinction behavior as well.

Neuronal activities in the lateral septum and lateral hypothalamus (Gonzalez-Lima et al., 1993) as well as the VTA (Kosobud et al., 1994) are modulated by rewarding aspects of ingestive behavior. The mammillary bodies, although implicated in supporting memory function (Sziklas and Petrides, 1998), also play a role in inhibiting punished responses (Shibata et al., 1986). During extinction, the withdrawal of expected rewards leads to a frustrative motivational state that has been well documented in rat pups in this specific task (Lobaugh et al., 1985; Amsel, 1992; Lilliquist et al., 1999). It is possible that the mammillary bodies, septum, lateral hypothalamus, and VTA play a role in evoking this frustrative motivational state. The ability to use frustrative cues to inhibit behavior during nonreward may require the hippocampal system, thus explaining the high interregional correlations within the hippocampus and between hypothalamus and VTA.

\section{Developmental changes in septohippocampal maturation}

The most significant finding in this study is the markedly different pattern of correlative activity in PSA12 pups, relative to the PSA17 group, during extinction training. One explanation for these differences may be the difference in speed of responding between age groups. As a test of this hypothesis, we measured glucose uptake in the primary motor cortex, a region known to change in glucose utilization with motor activity (EbrahimiGaillard et al., 1994). There were no net changes in glucose uptake in this region across groups. The only significant change in covariance patterns involving the primary motor cortex was its significant functional connection with the VTA in the $\mathrm{HC} 12$ group. Based on these data, there is no compelling evidence that the changes in covariance patterns across trained groups are related to running speed. 


\begin{tabular}{|c|c|c|c|c|c|c|c|c|c|c|}
\hline & EC & CA1 & CA3 & DG & MS & LS & MAM & SUBIC & VTA & PMC \\
\hline \multicolumn{11}{|l|}{ A. PSA17 } \\
\hline CA1 & 0.68 & & & & & & & & & \\
\hline CA3 & 0.56 & 0.91 & & & & & & & & \\
\hline DG & 0.56 & 0.98 & 0.96 & & & & & & & \\
\hline MS & 0.34 & 0.79 & 0.86 & 0.84 & & & & & & \\
\hline LS & 0.51 & 0.93 & 0.99 & 0.97 & 0.90 & & & & & \\
\hline MAM & 0.03 & 0.40 & 0.45 & 0.44 & 0.79 & 0.53 & & & & \\
\hline SUB & 0.59 & 0.97 & 0.90 & 0.97 & 0.88 & 0.93 & 0.50 & & & \\
\hline VTA & 0.26 & 0.78 & 0.93 & 0.88 & 0.92 & 0.95 & 0.56 & 0.84 & & \\
\hline PMC & 0.23 & 0.16 & 0.34 & 0.18 & 0.46 & 0.36 & 0.57 & 0.19 & 0.38 & \\
\hline LH & -0.14 & 0.42 & 0.44 & 0.49 & 0.76 & 0.51 & 0.87 & 0.53 & 0.59 & 0.12 \\
\hline \multicolumn{11}{|l|}{ B. $\mathrm{HC} 17$} \\
\hline CA1 & -0.35 & & & & & & & & & \\
\hline CA3 & 0.54 & 0.14 & & & & & & & & \\
\hline DG & 0.46 & 0.66 & 0.60 & & & & & & & \\
\hline MS & 0.81 & 0.20 & 0.78 & 0.81 & & & & & & \\
\hline LS & 0.86 & 0.11 & 0.66 & 0.77 & 0.98 & & & & & \\
\hline MAM & 0.89 & -0.13 & 0.46 & 0.54 & 0.88 & 0.94 & & & & \\
\hline SUB & 0.88 & -0.40 & 0.37 & 0.28 & 0.74 & 0.82 & 0.96 & & & \\
\hline VTA & 0.88 & -0.48 & 0.37 & 0.19 & 0.69 & 0.77 & 0.44 & 0.99 & & \\
\hline PMC & 0.74 & -0.39 & -0.27 & 0.66 & 0.66 & 0.81 & 0.86 & 0.81 & 0.77 & \\
\hline LH & 0.79 & -0.63 & 0.26 & -0.19 & 0.53 & 0.62 & 0.82 & 0.95 & 0.98 & 0.73 \\
\hline
\end{tabular}

Reliably significant correlations are bold.

\begin{tabular}{|c|c|c|c|c|c|c|c|c|c|c|}
\hline & $\mathrm{EC}$ & CA1 & CA3 & DG & MS & LS & MAM & SUBIC & VTA & PMC \\
\hline \multicolumn{11}{|l|}{ A. PSA12 } \\
\hline CA1 & 0.89 & & & & & & & & & \\
\hline CA3 & 0.65 & 0.68 & & & & & & & & \\
\hline DG & 0.77 & 0.76 & 0.77 & & & & & & & \\
\hline MS & 0.53 & 0.85 & 0.48 & 0.59 & & & & & & \\
\hline LS & 0.74 & 0.86 & 0.70 & 0.94 & 0.81 & & & & & \\
\hline MAM & -0.29 & -0.15 & 0.05 & 0.20 & 0.17 & 0.23 & & & & \\
\hline SUB & 0.85 & 0.94 & 0.71 & 0.81 & 0.84 & 0.89 & 0.08 & & & \\
\hline VTA & 0.29 & 0.51 & 0.47 & 0.68 & 0.65 & 0.78 & 0.57 & 0.51 & & \\
\hline PMC & 0.40 & 0.61 & 0.53 & 0.73 & 0.63 & 0.81 & 0.09 & 0.50 & 0.80 & \\
\hline LH & 0.07 & 0.08 & 0.17 & -0.07 & 0.36 & 0.10 & 0.83 & 0.24 & 0.76 & 0.36 \\
\hline \multicolumn{11}{|l|}{ B. $\mathrm{HC} 12$} \\
\hline CA1 & 0.04 & & & & & & & & & \\
\hline CA3 & 0.01 & 0.69 & & & & & & & & \\
\hline DG & 0.23 & 0.42 & 0.85 & & & & & & & \\
\hline MS & 0.56 & 0.17 & 0.550 & 0.39 & & & & & & \\
\hline LS & 0.31 & 0.08 & 0.01 & -0.35 & 0.69 & & & & & \\
\hline MAM & 0.84 & -0.16 & 0.24 & 0.41 & 0.82 & 0.38 & & & & \\
\hline SUB & 0.47 & 0.40 & 0.59 & 0.89 & 0.21 & -0.48 & 0.43 & & & \\
\hline VTA & 0.71 & 0.02 & 0.44 & 0.44 & 0.96 & 0.56 & 0.95 & 0.34 & & \\
\hline PMC & 0.71 & 0.81 & 0.85 & 0.71 & 0.94 & 0.82 & 0.96 & 0.89 & 0.99 & \\
\hline LH & 0.24 & 0.41 & -0.05 & 0.30 & 0.49 & 0.57 & 0.48 & 0.39 & 0.51 & 0.65 \\
\hline
\end{tabular}

Reliably significant correlations are bold.

Alternatively, the changes in functional relationships more likely reflect developmental differences in the functional integrity of the network of regions sampled, particularly that of the hippocampal system. For example, the granule cells of the dentate gyrus are still proliferating at a substantial rate at P12 (Schlessinger et al., 1975). Although mossy fibers, the axonal projections of granule cells to CA3 pyramidal cells, are present at P12 their density is far greater at the older age (Amaral and Dent, 1981).
The "thorny excrescences", while relatively adult-like by P18, only begin to emerge at $\sim$ P11 (Amaral and Dent, 1981). Furthermore, the physiology of various hippocampal cell types are likely to be altered, because the receptor composition and sensitivity, for example, among GABA $_{\mathrm{A}}$ (Fritschy et al., 1994) and NMDA receptors (Bowe and Nadler, 1990) are different at P12. In light of these data, the propensity for concerted functional activity within the hippocampal system should be reduced at $\mathrm{P} 12$ relative to $\mathrm{P} 17$ 


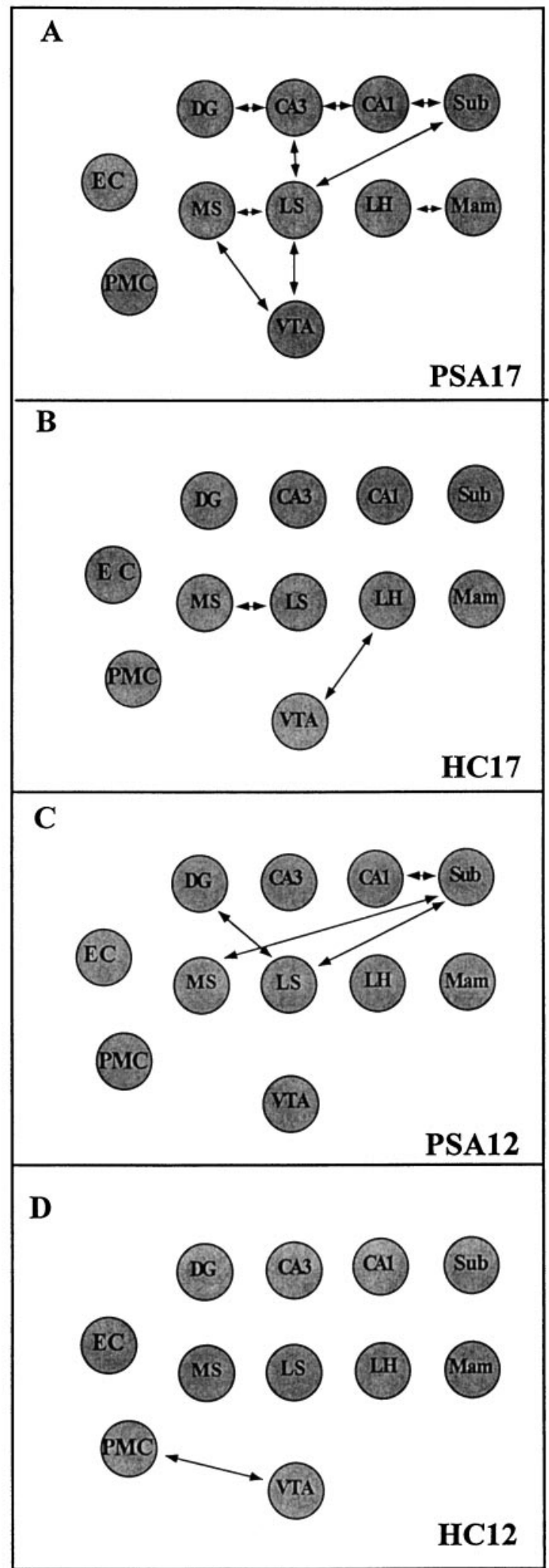

Figure 7. Covariance patterns of FDG uptake across groups. Black arrows indicate reliable pairwise correlations significantly different from zero $(p<0.05)$. Note changes in covariance patterns between PSA $17(A)$ and PSA12 $(C)$. The same correlations did not appear between handled control groups $(B, D)$, indicating the altered covariance patterns across PSA groups were related to extinction training rather than handling or developmental effects.
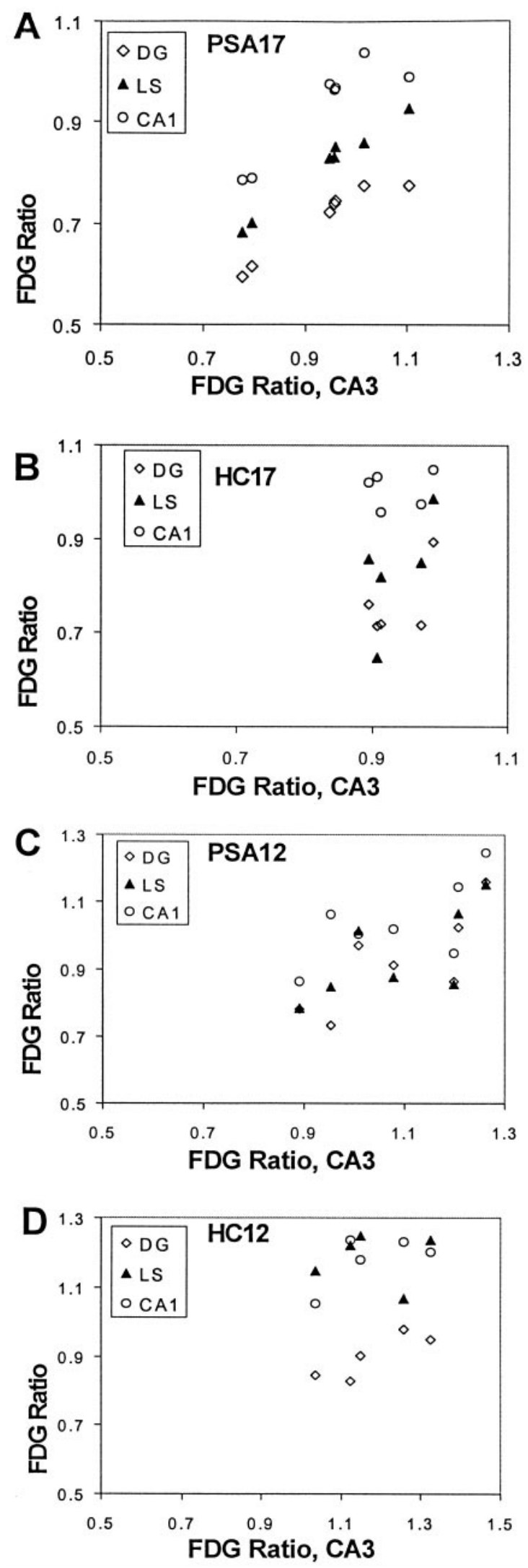

Figure 8. Scatter plots of correlations between CA3 and dentate gyrus $(D G), \mathrm{CA} 3$ and lateral septum $(L S)$, and CA3 and CA1. FDG ratio, or whole brain ratio, is the raw FDG value divided by the whole brain average. Note the linearity of values in the PSA17 group $(A)$ relative to those found within $\mathrm{HC} 17(B)$, PSA12 $(C)$, and HC12 $(D)$ groups. 


\begin{tabular}{lc}
\hline $\begin{array}{l}\text { Table 4. Brain behavior correlations between FDG regional activity and } \\
\text { extinction rate }\end{array}$ \\
Region of interest & Correlation $(r$ value $)$ \\
\hline Entorhinal cortex & 0.40 \\
CA1 & $0.71^{*}$ \\
CA3 & $0.70^{*}$ \\
DG & 0.38 \\
Subiculum & $0.69^{*}$ \\
Medial septum & $0.64^{*}$ \\
Lateral septum & $0.60^{*}$ \\
Lateral hypothalamus & 0.30 \\
Mammillary bodies & $0.68^{*}$ \\
Ventral tegmental area & 0.46 \\
Primary motor cortex & -0.03
\end{tabular}

*Significantly different from zero; $p<0.05$.

pups, and any behavior that is mediated by the system should thus be compromised. Given that a major difference between PSA12 and PSA17 animals is the absence of reliable correlations between CA3 and the dentate gyrus, CA1, and subiculum in the PSA12 group, this prediction is supported by the data. Taken together, the functional immaturity of the hippocampal system at P12, which appears to be reflected in the covariance analysis, may underlie the attenuated extinction rates in these animals. Furthermore, it is possible that the $\mathrm{P} 12$ rats were deficient in adjusting to the change in experimental contingencies between PSA acquisition and extinction, since the hippocampus has been implicated in such a function (Diaz-Granados et al., 1992).

Although septohippocampal (Linke and Frotscher, 1993) and hippocamposeptal (Linke et al., 1995) connections are well established by P12, information flow between the septum and hippocampus is still likely to be altered at P12 because of the functional immaturity within the hippocampal formation. It is possible that the absence of the intraseptal and septal-VTA correlations in the P12 group may arise for this reason. This is supported to some extent by the covariance patterns found in the PSA17 group: the high positive intrahippocampal correlations (i.e., between dentate gyrus, CA3, CA1, and subiculum) and septohippocampal functional couplings (i.e., subiculum and CA3 to the lateral septum) coincide with the appearance of the intraseptal and septal-VTA functional connections (Fig. 7A). Because correlations do not imply causal influences, these issues merit further investigation using extensions of the covariance analysis, such as structural equation modeling (McIntosh and GonzalezLima, 1994), as well as other neurobiological approaches.

In conclusion, the ontogenetic changes in mean FDG uptake suggest that the functional properties of the mammillary bodies, CA1, CA3, subiculum, medial septum, and VTA change between $\mathrm{P} 12$ and P17. This appears to be the case because the covariance patterns between these and the rest of sampled regions changed markedly between the P12 and P17 PSA groups during extinction training. Taken together, the behavioral and neural results suggest that septohippocampal and mesencephalic circuits may be functionally dissociated at P12, and the subsequent maturation of functional connectivity between these circuits allows for the more rapid expression of behavioral inhibition during extinction at P17.

\section{REFERENCES}

Amaral DG, Dent JA (1981) Development of the mossy fibers of the dentate gyrus: a light and electron microscopic study of the mossy fibers and their expansions. J Comp Neurol 195:51-86.
Amsel A (1992) Frustration theory. New York: Cambridge UP.

Bowe MA, Nadler JV (1990) Developmental increase in the sensitivity to magnesium of NMDA receptors on CA1 hippocampal pyramidal cells. Dev Brain Res 56:55-61.

Bronstein PM, Neiman H, Wolkoff FD, Levine MJ (1971) The development of habituation in the rat. Anim Learn Behav 2:92-96.

Chugani HT, Phelps ME (1986) Maturational changes in cerebral function in infants determined by [18]FDG positron emission tomography. Science 231:840-843.

Chugani HT, Hovda DA, Villablanca JR, Phelps ME, Xu WF (1991) Metabolic maturation of the brain: a study of local cerebral glucose utilization in the developing cat. J Cereb Blood Flow Metab 11:35-47.

Diaz-Granados JL, Greene PL, Amsel A (1992) Learned persistence in the infant rat is affected by $\mathrm{x}$-irradiation-induced hippocampal granulecell hypoplasia. Behav Neural Biol 61:251-259.

Ebrahimi-Gaillard A, Beck T, Wree A, Roger M (1994) Metabolic mapping of the forelimb motor system in the rat: local cerebral glucose utilization following execution of forelimb movements mainly involving proximal musculature. Somatosens Mot Res 11:229-241.

Feigley DA, Parsons PA, Hamilton LW, Spear NE (1972) Development of habituation to novel environments in the rat. J Comp Physiol Psychol 79:443-452.

Fritschy J-M, Paysan J, Enna A, Mohler H (1994) Switch in the expression of rat GABAa-receptor subtypes during postnatal development: an immunohistochemical study. J Neurosci 14:5302-5324.

Gonzalez-Lima F (1992) Brain imaging of auditory learning functions in rats: studies with fluorodeoxyglucose autoradiography and cytochrome oxidase histochemistry. In: Advances in metabolic mapping techniques for brain imaging of behavioral and learning functions (Gonzalez-Lima F, Finkenstadt TH, Scheich H, eds), pp 39-109. Boston: Kluwer Academic.

Gonzalez-Lima F, Helmstetter FJ, Agudo J (1993) Functional mapping of the rat brain during drinking behavior: a fluorodeoxyglucose study. Physiol Behav 54:605-612.

Henke PG (1977) Dissociation of the frustration effect and the partial reinforcement extinction effect after limbic lesion in rats. J Comp Physiol Psychol 91:1032-1038.

Hochberg Y (1988) A sharper Bonferroni procedure for multiple tests of significance. Biometrics 75:800-802.

Horwitz B, Grady CL, Schlageter RD, Rapoport SI (1987) Intercorrelations of regional cerebral glucose metabolic rates in Alzheimer's disease. Brain Res 407:294-306.

Horwitz B, Grady CL, Haxby JV, Schapiro MB (1992a) Functional associations among human posterior extrastriate brain regions during object and spatial vision. J Cognit Neurosci 4:311-322.

Horwitz B, Soncrant TT, Haxby JV (1992b) Covariance analysis of functional interactions in the brain using metabolic and blood flow data. In: Advances in metabolic mapping techniques for brain imaging of behavioral and learning functions (Gonzalez-Lima F, Finkenstadt TH, Scheich H, eds), pp 189-212. Boston: Kluwer Academic.

Jarrad LE, Feldon J, Rawlins JNP, Sinden JD, Gray JA (1986) The effects of intrahippocampal ibotenate on resistance to extinction after continuous or partial reinforcement. Exp Brain Res 61:519-530.

Kimble DP, Kimble RJ (1965) Hippocampectomy and response perseveration in the rat. J Comp Physiol Psychol 60:474-476.

Kosobud AE, Harris GC, Chapin JK (1994) Behavioral associations of neuronal activity in the ventral tegmental area of the rat. J Neurosci 14:7117-29.

Lilliquist MW, Nair HP, Gonzalez-Lima F, Amsel A (1999) Extinction after regular and irregular reward schedules in the infant rat: influence of age and training duration. Dev Psychobiol 34:57-70.

Linke R, Frotscher M (1993) Development of the rat septohippocampal projection: tracing with DiI and electron microscopy of identified growth cones. J Comp Neurol 332:69-88.

Linke R, Pabst T, Frotscher M (1995) Development of the hippocamposeptal projection in the rat. J Comp Neurol 351:602-616.

Lobaugh NJ, Bootin M, Amsel A (1985) Sparing of patterned alternation but not partial reinforcement effect after infant and adult hippocampal lesions in the rat. Behav Neurosci 99:46-59.

McIntosh AR, Gonzalez-Lima F (1994) Network interactions among limbic cortices, basal forebrain, and cerebellum differentiate a tone conditioned as a Pavlovian excitor or inhibitor: fluorodeoxyglucose 
mapping and covariance structural equation modeling. J Neurophysiol 72:1717-1733.

Myslivecek J, Hassmannova J (1991) Step-down passive avoidance in the rat ontogeny. Acta Neurobiologiae Experimentalis 51:89-96.

Nehlig A, de Vasconceles AP, Boyet S (1989) Quantitative autoradiographic measurement of local cerebral glucose utilization in freely moving rats during postnatal development. J Neurosci 8:2321-2333.

Rawlins JNP, Feldon J, Gray JA (1980) The effects of hippocampectomy and of fimbria section upon the partial reinforcement effect in rats. Exp Brain Res 38:273-283.

Schlessinger AR, Cowan WM, Gottlieb DI (1975) An autoradiographic study of the time origin and the pattern of granule cell migration in the dentate gyrus of the rat. J Comp Neurol 159:149-176.
Sherwood NM, Timiras PS (1970) A stereotaxic atlas of the developing rat brain. Berkeley: University of California.

Shibata K, Kataoka Y, Yamashita K, Ueki S (1986) An important role of the central amygdaloid nucleus and mammillary body in the mediation of conflict behavior in rats. Brain Res 372:159-162.

Sokoloff L (1992) Imaging techniques in studies of neural functions. In: Advances in metabolic mapping techniques for brain imaging of behavioral and learning functions (Gonzalez-Lima F, Finkenstadt TH, Scheich H, eds), pp 1-31. Boston: Kluwer Academic.

Sziklas V, Petrides M (1998) Memory and the region of the mammillary bodies. Prog Neurobiol 54:55-70.

Winocur G, Mills JA (1969) Hippocampus and septum in response inhibition. J Comp Physiol Psychol 67:353-357. 